\title{
LA TRANSICIÓN PALEOLÍTICA MEDIO-SUPERIOR EN LA REGIÓN CENTRO-ORIENTAL DE LA CORNISA CANTÁBRICA
}

\section{The Transition Middle Palaeolithic-Upper Palaeolithic in the centre-east region of the Cantabrian Region}

\author{
Federico Bernaldo de Quirós, Álvaro Arrizabalaga, \\ José Manuel Maíllo-Fernández y María José Iriarte*
}

Recibido el 12 de febrero de 2008. Aceptado el 15 de marzo de 2008

Resumen. Con este trabajo, en homenaje al profesor Eduardo Ripoll Perelló, queremos presentar algunos de los datos más recientes sobre la transición entre el Paleolítico medio y el superior en la región centro-oriental de la cornisa cantábrica. Para ello, tomamos como referencia las investigaciones de cuevas como El Castillo y Cueva Morín en Cantabria y Lezetxiki y Labeko Koba en el País Vasco. Por último, presentamos una serie de reflexiones en relación con algunos aspectos de dicha transición.

Palabras clave: Transición, Paleolítico medio, Paleolitico superior, Auriñaciense, Musteriense, Auriñaciense de Transición, Chatelperroniense, Cantábrica.

Abstract. In this paper, which is a tribute to Professor Eduardo Ripoll, we want to present some results related to the Middle-Upper Paleolithic Transition in the mid-east Cantabrian Region. Our reference sites are El Castillo and Cueva Morin in Cantabria and Lezetxiki and Labeko Koba in the Basque Country. Finally, we present some reflections concerning to some issues of the Middle-Upper Paleolithic Transition.

Key Words: Transition, Middle Palaeolithic, Upper Palaeolithic, Aurignacian, Mousterian, Aurignacian of Transition, Chatelperronian, Cantabrian Iberia.

\section{LAS ÚLTIMAS INVESTIGACIONES DE CAMPO EN EL MEDIO CANTÁBRICO}

El objetivo planteado con este texto es dar una visión actualizada sobre algunos de los trabajos de campo y laboratorio que afectan a la transición entre el Paleolítico medio y el superior en la zona centro-oriental de la cornisa cantábrica. Tratándose de un momento especialmente conflictivo y sometido a permanente debate, preferimos dar prioridad a la presentación de los sitios y a su información, dedicando una atención más secundaria a la discusión de tales aspectos, en la medida que en ella expresaremos con mayor frecuencia opiniones.
Los últimos quince años de la investigación han generado para el área mencionada un copioso volumen de información, tanto en forma de materiales arqueológicos, como de dataciones, analíticas y sintesis (Cabrera et al., 2004). Esto guarda directa relación con el interés suscitado por la Transición entre Paleolítico medio y superior (asumiendo el cambio filético entre el tipo humano neandertal y cromagnon) en esta región, en la que se puede constatar un aparente solapamiento cronológico entre las circunstancias de todo tipo, supuestamente caracteristicas de ambas especies. En este sentido, consideramos importante presentar algunas de las novedades que se han registrado en el trabajo de campo durante los últimos años en la zona de estudio.

(*) Federico Bernaldo de Quirós: Área de Prehistoria, Universidad de León. Campus de Vegazana, León, decfbq@unileon.es Álvaro Arrizabalaga y María José Iriarte: Grupo de Investigación Consolidado y de Alto Rendimiento de Prehistoria de la Universidad del País VasCO (9/UPV 155.130-14570/2002).

José Manuel Maillo-FernÁndez: Dpto. de Prehistoria y Arqueología, UNED. C/ Senda del rey, 7. 28040 Madrid, jlmaillo@geo.uned.es 


\subsection{La Cueva del Castillo (Puente Viesgo, Cantabria)}

La Cueva del Castillo fue descubierta por Alcalde del Río en 1903. A partir de 1910, H. Obermaier y P. Wernert, financiados por el Institut de Paléontologie Humaine de París, comenzaron la excavación principal que continuó hasta 1914, cuando el inicio de la Primera Guerra Mundial obligó a suspender los trabajos. En 1973, V. Cabrera retoma los estudios sobre el yacimiento, recopilando y publicando (Cabrera, 1984) la documentación inédita y los materiales recogidos por Obermaier, al mismo tiempo que se reiniciaron las excavaciones arqueológicas en el yacimiento en 1980 con un equipo interdisciplinar, dirigido por ella misma y uno de nosotros (Cabrera y Bernaldo de Quirós, 2000). Estos trabajos han ofrecido un marco cronológico contrastado a partir de dataciones por diversos métodos físico-químicos, como el AMS, el ESR y el $T h / U$, que nos sitúan estos niveles de la ocupación humana entre los 150.000 y los 30.000 años, antes del presente. Presenta así ocupaciones del Paleolítico Medio -unidades 26 a 20-, todo el Paleolítico Superior con niveles del Auriñaciense de Transición (niveles $18 b$ y 18c), Auriñaciense arcaico (nivel 16), Gravetiense (unidades 14 y 12), Solutrense (Unidad 10), Magdalenienses (Unidades 8 y 6 ) y Aziliense (Unidad 4).

Las investigaciones recientes se han centrado en el área exterior del vestíbulo, abordándose especialmente los niveles relacionados con los inicios del Paleolítico Superior y el Musteriense, que ya han sido objeto de una completa serie de dataciones. La unidad 20, Musteriense, ha sido dividida en cuatro niveles $20 \mathrm{a} / \mathrm{b}, 20 \mathrm{c}, 20 \mathrm{~d}$ y $20 \mathrm{e}$. A partir de las excavaciones de Obermaier, todas ellas caracterizadas por la presencia de un Musteriense Charentiense con hendedores (Cabrera, 1983). Por su parte, el estudio de la nueva colección matiza estas conclusiones, clasificando el nivel 20e como Musteriense típico (Sánchez Fernández, 2005). En ellos encontramos, junto a las características cadenas operativas discoides, la presencia de una cadena operativa de producción de hojitas que implica importantes relaciones de continuidad con los niveles del Auriñaciense de Transición que se sitúan sobre ellos. En uno de los niveles encontramos al menos dos hogares con concentraciones de carbón en 20c1 y 20c3. Debajo se sitúa el nivel 21, cuyas características están aún en estudio, pero en el que apareció un canto decorado de cuarcita fina gris, del mismo tipo utilizado en la producción de herramientas (Tipo C1 de Cabrera et al., 1996a; Cabrera et al., 2000: 87).

La unidad 18 se corresponde con la reocupación de la cueva tras un derrumbe que selló la Unidad 20 y que formó la Unidad 19. En este momento se han descubierto evidencias que han puesto de relieve la importancia de este yacimiento en los procesos de transición hacia el Paleolítico Superior (Cabrera-Valdés et al., 2005). Esta unidad se divide en dos niveles que presentan actividades distintas. El nivel 18c es el nivel más antiguo de los cazadores recolectores del Paleolítico Superior, y presenta abundantes restos de carbón en una zona donde los grupos que habitaron la cueva barrieron sus hogueras para realizar otras nuevas. Además, podemos deducir que hace 40.000 años tenían una caza especializada en el ciervo. De estos animales aprovecharían el asta como materia prima para realizar puntas de azagayas. La tecnología de la talla de los útiles líticos presenta esquemas operativos laminares, algunos muy similares a los que se encuentran en el Auriñaciense arcaico (Lloret y Maillo-Fernández, 2006). Se documenta también la decoración de utensilios de uso cotidiano con marcas simbólicas (Cabrera et al., 2001). El nivel $18 \mathrm{~b}$, por su parte, tiene una formación ligeramente posterior fechada hacia el 38.500 B.P., en la que se mantienen pautas parecidas al anterior en la tecnología lítica y en la caza, aunque difiere en la actividad realizada en el área excavada (descuartizamiento de animales cazados) y una intensificación de las manifestaciones simbólicas, iniciándose el arte figurativo sobre elementos mobiliares (Cabrera et al., 2006).

La industria hallada en el nivel 16, datada en $34.300 \pm$ 1.000 (GifA-95539), debe ser clasificada como Auriñaciense arcaico o Protoauriñaciense. Aunque la colección es muy exigua, se encuentran entre el material lítico las hojitas Dufour como elemento tipológico más numeroso y característico. La producción de hojitas se realiza a partir de núcleos prismáticos unipolares y raspadores carenados. Apareció el fragmento distal de una azagaya.

\subsection{Cueva Morín}

Cueva Morín se encuentra en Villanueva de Villaescusa (Cantabria), a unos nueve kilómetros de la ciudad de Santander. Fue descubierta por H. Obermaier y P. Wernert en 1910 y excavada por J. Carballo y el conde de la Vega del SeIla entre 1917 y 1919 (Vega del Sella, 1921; Carballo, 1923). La última intervención arqueológica en el yacimiento fue llevada a cabo por un equipo hispano-estadounidense dirigido por J. González Echegaray y L. G. Freeman (González Echegaray y Freeman, 1971, 1973 y 1978). Estudios posteriores sobre la industria lítica han sido realizados por A. Arrizabalaga (1995) para los inicios del Paleolítico superior y J. M. Maillo (2003) para la Transición entre el Paleolítico medio y el superior. Cueva Morín representa una de las estratigrafías clave para el conocimiento del Paleolítico medio y superior en la región Cantábrica. En ella están representados 22 niveles que abarcan desde el Musteriense hasta el Aziliense.

En cuanto a la Transición entre el Paleolítico medio y el superior y a las primeras etapas del Auriñaciense disponemos de los materiales concernientes a siete niveles arqueológicos: dos correspondientes al Musteriense final (12 y 11), uno Chatelperroniense (10); dos pertenecientes al Protoauriñaciense o Auriñaciense arcaico (9 y 8) y dos correspondientes al Auriñaciense antiguo (7 y 6). 
El yacimiento presenta dos series de dataciones. En la primera de ellas se obtuvieron unas dataciones cuyos resultados parecen incoherentes entre sí, siendo, como norma general, fechas más recientes de las normalmente admitidas para estos momentos históricos (Stuckenrath, 1978). Recientemente se han obtenidos dos nuevas dataciones para los niveles 11 y 8 que han envejecido sensiblemente los niveles anteriormente datados (Maíllo et al., 2001).

El Musteriense final (niveles 11 y 12) se caracteriza desde un punto de vista tecnológico por los esquemas de concepción discoide bajo dos métodos: unifacial y bifacial. Las materias primas son variadas, pero destacan el sílex y la cuarcita de grano fino y los soportes se destinan a la elaboración de denticulados y raederas. Existe un esquema operativo laminar orientado a la obtención de hojitas a partir de núcleos prismáticos bipolares. Tipológicamente, el conjunto está dominado por los denticulados $y$, en menor número, por las raederas. Corresponderian a un Musteriense de denticulados sensu Bordes. El nivel 11 ha sido recientemente datado obteniéndose una fecha de $39.770 \pm 730$ B.P. (GifA-96264) (Maillo et al., 2001).

El Chatelperroniense de Cueva Morín también está dominado por los esquemas de lasca tipo discoide similares a los del Musteriense. Aunque el componente laminar es, cualitativamente, más relevante. Existen dos esquemas de producción de hojas. El más abundante es el esquema operativo realizado a partir de núcleos prismáticos unipolares. El otro esquema operativo es el que emplea núcleos prismáticos bipolares. Los soportes obtenidos en este último método se destinan a la elaboración de puntas de Chatelperrón. No existe una producción específica de hojitas. El Chatelperroniense de Cueva Morin se caracteriza por un bajo número de puntas de Chatelperron $(4,2 \%)$, un mayor número de raspadores que de buriles y un amplio porcentaje de piezas de sustrato.

El Auriñaciense arcaico está presente en los niveles 9 y 8. Tecnológicamente, se caracterizan por un fuerte desarrollo de los esquemas operativos de producción de hojitas a partir de

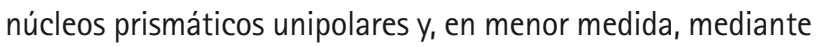
núcleos de tipo raspador carenado, existiendo un continuum entre la producción de hojas y de hojitas. Es también importante la producción de lascas mediante esquemas de tipos discoide. Tipológicamente se define por un alto porcentaje de hojitas Dufour (17,9\% en el nivel 8). Destacan también las piezas con retoque lateral y de fondo común, siendo los raspadores más abundantes que los buriles. El nivel 8 presenta una reciente datación de $36.590 \pm 770$ B.P. (GifA-96263).

En el Auriñaciense antiguo, desde un punto de vista tecnológico, se produce una disociación entre la producción de hojas y de hojitas. Las hojas se producen a partir de núcleos prismáticos unipolares y para las hojitas se emplean esquemas operativos de tipo raspador carenado. La producción de lascas es más limitada y orientada, en gran medida, a la obtención de lascas para soportes de núcleos carenados. Tipo- lógicamente, los raspadores destacan sobre los buriles, sobre todo los raspadores espesos. La industria ósea es escasa y fragmentaria. En el nivel 7 disponemos de dos fragmentos de azagaya de sección cuadrangular y otros dos de sección circular, además de un canino perforado de ciervo. Por su parte, en el nivel 6 tenemos un fragmento basal de azagaya, seis azagayas (cuatro de sección circular, una subcuadrangular y otra subtriangular) y una varilla.

\subsection{Labeko Koba (Arrasate, País Vasco)}

El yacimiento de Labeko Koba, cuya primera cita remonta hasta 1972 (Altuna et al., 1995) prácticamente se descubre y excava entre 1987 y 1989, bajo la dirección de A. Arrizabalaga (Arrizabalaga y Altuna, 2000). Siendo la monografía del yacimiento tan próxima en el tiempo, sólo procede remitir a la misma y recordar, brevemente, la estratigrafía del yacimiento. Labeko Koba incluye un depósito ininterrumpido de materiales arqueológicos y sedimentos desde el Castelperroniense (nivel IX inferior), hasta el Auriñaciense antiguo avanzado (nivel IV).

Tenemos constancia de unas primeras visitas a la cueva por parte de las especies que rivalizarán por ocuparla (carnívoros y humanos) desde la base de la secuencia (nivel IX inferior). La presencia humana, muy ocasional, puede interpretarse en relación con el aprovisionamiento de recursos bióticos (carne, piel, cuerna, hueso, etc.) presentes en la sima por la actividad de los carnívoros, principales actores en la tafocenosis de la base del depósito. Las primeras fases de colmatación de Labeko Koba, que suponen prácticamente la mitad de la potencia arqueológica total del yacimiento, constituyen el nivel IX (en algunos cuadros se aproxima a los dos metros de espesor). El subnivel IX inferior ha sido atribuido al Castelperroniense, fundamentalmente por el peso que tiene sobre un lote restringido de industria lítica la presencia de tres puntas de Châtelperron. También está presente un fragmento de azagaya, muy deteriorada. El tramo superior del nivel IX ocupa la mayor parte de la unidad y carece prácticamente de restos de industria (apenas cinco lascas de sílex y otros tantos posibles percutores en asta de megaceros). Desde el punto de vista cultural resulta complicado etiquetar este subnivel, que se ubica entre el Castelperroniense (IX inferior) y el Protoauriñaciense (VII), separándolos más netamente que el irregular nivel VIII.

En cuanto al nivel VII, marca una inflexión clara con respecto a los niveles subyacentes. Por primera vez en la secuencia de Labeko Koba, el ser humano cobra protagonismo. En particular por la composición de su industria lítica, este nivel se conforma como claramente Protoauriñaciense, en concreto de la variedad con abundantes laminitas de retoque semiabrupto (tipo Dufour). En el nivel VII han sido recuperados varios restos (un canto con líneas grabadas, una pequeña bola de ámbar y varias evidencias de decoración sobre soporte óseo) que testimonian que, al menos desde el 
Protoauriñaciense, estos grupos disponían de un universo simbólico similar al que se observará a lo largo del Paleolítico superior.

Los niveles VII y V se encuentran separados por un nivel $\mathrm{VI}$, muy pobre, que parece bascular culturalmente más hacia el nivel V que hacia el VII. Aunque la industria lítica no es significativa, en este nivel existe una azagaya de base hendida, testimonio que nos acota la ocupación como una primera presencia de gentes del Auriñaciense antiguo. La adscripción cronológica del nivel $V$ también es segura, a la vista de la existencia de una azagaya de base hendida casi completa. Los tecnocomplejos líticos apuntan en la misma dirección, al presentarse los niveles modal y grupal dentro de los parámetros observados para el Auriñaciense antiguo en otros niveles del Cantábrico (Cueva Morín o la Cueva del Polvorín, entre otros).

El nivel IV de Labeko Koba se presenta más problemático de datar. Nuestro principal apoyo para la adscripción del nivel IV consiste en su fuerte similitud con el nivel $\mathrm{V}$, a todos los efectos (ambiental, sedimentario, industrial, comportamental). Si observamos el registro lítico, comprobamos que ambos niveles tienden a asociarse. La estructura lítica más diagnóstica a efectos culturales (la modal) muestra una gran similitud entre ambos modelos, a pesar de que el retoque semiabrupto, todavía fuertemente presente en el nivel V casi desaparece en el nivel IV. También resulta fácil referir este modelo de Auriñaciense antiguo con otros posibles paralelos (unidades de Gatzarria o Cueva Morín). La industria ósea no aporta informaciones determinantes a efectos de cronología cultural. En consecuencia, consideraremos a este nivel IV como una fase más avanzada del Auriñaciense antiguo. Debe de precisarse en este punto que no nos referimos a un Auriñaciense evolucionado o évolué en su nomenclatura francesa.

\subsection{Lezetxiki (Arrasate, País Vasco)}

La Carta Arqueológica de Guipúzcoa (Altuna et al., 1995) sitúa en 1927 el descubrimiento del yacimiento, por parte de Justo Jáuregui. La excavación del depósito dio comienzo en 1956, prolongándose en sucesivas campañas anuales hasta 1968. En las campañas de excavación 1956-1968 se recuperaron materiales, muestras e información contextual de una amplia superficie (unos 75 metros cuadrados de media). Esta superficie fue sometida a un tratamiento muy avanzado en el momento en el que se desarrolló la excavación clásica (micro y grandes mamíferos, sedimentología, un primer ensayo palinológico y una datación de $\mathrm{C}^{14}$ ). La actuación dirigida por uno de nosotros desde 1996, resulta mucho más limitada. Trabajamos en una superficie de unos diez metros cuadrados en el cantil sur de Lezetxiki, con lo que difícilmente contaremos con nuevos materiales que obliguen a matizar lo que conocíamos con anterioridad acerca de esta secuencia. Nuestro esfuerzo por recuperar informaciones novedosas de la secuencia de Lezetxiki pretendía poner en valor un depósito clásico e intentar resolver algunas cuestiones que, entendíamos, quedaban pendientes de aclaración desde el cierre de las anteriores campañas. Igualmente, venimos desarrollando sucesivas campañas de excavación en Lezetxiki II, con el objetivo de proporcionar un contexto estratigráfico adecuado al conocido húmero de Leibar.

Los niveles de "transición" entre el Paleolítico medio y el superior en Lezetxiki son, básicamente, los niveles III y IV (este último, con dos subniveles fértiles, IVa y IVc). Incluyen un lote significativo de industria lítica, pero prácticamente ningún resto de industria ósea. Desde una perspectiva tecnotipológica y de gestión de materias primas, estas tres unidades presentan características ambivalentes, propias tanto del final del Musteriense, como del inicio del Paleolítico superior. Las excavaciones antiguas parecian dar indicaciones en esta dirección, que han sido reiteradamente rechazadas por sospecharse la remoción de estos niveles o un trabajo de campo poco cuidadoso. Sin embargo, nuestra intervención proporciona la misma lectura, permitiendo descartar las alteraciones posdeposicionales de este grueso paquete sedimentario (que supera el metro de potencia en varias zonas). En nuestra opinión contamos con un registro de la Transición Paleolítico medio-superior poco común y de difícil interpretación, más aún en ausencia de dataciones absolutas. Pero su interpretación obliga a pensar en clave cultural y no sólo estratigráfica, según nuestra opinión. Entre los elementos que motivan que el debate sobre la cualificación y estudio de estos niveles sea tan interesante incluiremos la presencia de restos fósiles de neandertal en la base del nivel III, la aparición de restos malacológicos de uso ornamental en el nivel III y IVc o la presencia simultánea de cadenas operativas Levallois y microlaminares, al menos en las unidades del nivel IV (Arrizabalaga, 2006).

\section{ESTADO DE LA CUESTIÓN EN ALGUNAS CUESTIONES CLAVE DE LA INVESTIGACIÓN}

Procedemos a continuación a hacer una breve perspectiva acerca del estado de la cuestión, en el dominio geográfico ubicado entre la Cueva de El Castillo y Brassempouy.

\subsection{Cronología}

Tratar la cronología de la Transición entre el Paleolítico medio y el superior resulta una labor cuyos resultados han de ser tomados de manera cautelosa. En primer lugar, tenemos la limitación del propio método de datación, generalmente el $\mathrm{C}^{14}$, el cual tiene su límite de fiabilidad en estos momentos. La segunda cuestión es de índole arqueológica: ¿dónde establecemos el límite del Musteriense Final? 0 ¿en qué punto exacto ubicamos las primeras evidencias del Paleolítico superior? Sin duda, todo ello, unido a la propia idiosincrasia 
de este momento (la Transición) complica enormemente el cuadro de estudio.

El final del Musteriense lo hemos ubicado en torno al 50-39.000 B.P. a partir de los resultados obtenidos en El Castillo y Cueva Morín (Cabrera y Bischoff, 1989; Cabrera et al., 1996b; Maíllo Fernández et al., 2001), aunque existen yacimientos cuyas dataciones son bastante más recientes como El Esquilleu (Baena et al., 2006). Solapándose con el Musteriense final encontramos, por un lado, el Auriñaciense de Transición datado en 40.000 B.P. para el nivel 18c y 38.500 B.P. para el $18 \mathrm{~b}$ en El Castillo y el Chatelperroniense, el cual estratigráficamente, se ubica por debajo del Auriñaciense arcaico en Cueva Morín y Labeko Koba, aunque aquí la datación $\mathrm{C}^{14}$ resulta muy reciente: $34.215 \pm 1.265$ B.P. (UA-3324). El Auriñaciense arcaico se comienza a datar en la zona vasco-cantábrica en torno al 36.500 B.P. en yacimientos como La Viña en Asturias (Fortea, 1995), Cueva Morín, El Castillo, Labeko Koba e Isturitz (Maillo et al., 2001; Cabrera et al., 2004; Arrizabalaga, 2000a; Normand, 2002) prolongándose hasta el 30.000 B.P.

Por tanto, revisando las diferentes fechas radiométricas en torno a la Transición, podemos observar que existe cierto solapamiento de las mismas, por ejemplo el Chatelperroniense de Labeko Koba y el Auriñaciense arcaico de Cueva Morín. Este hecho puede responder a dos causas: una cultural y otra física. La cultural respondería a la posibilidad de que conviviesen diferentes tecnocomplejos en esta región durante este intervalo temporal, bien porque respondiesen a tradiciones culturales diferentes o bien a facies económicas dentro de un tecnocomplejo mayor. No entramos a valorar aquí el tipo humano autor de cada uno de los conjuntos. Más aún cuando la evidencia del registro fósil no permite aventurar datos completos. La segunda hipótesis, de carácter físico está ligada a la propia naturaleza de los métodos radiométricos de datación. $\mathrm{El}^{\mathrm{C}^{14}}$ está al límite de su uso con garantías y su correlación con otros métodos ( $T L, E S R$, sobretodo) no parece aún clara. Por lo que cabe la posibilidad de que este la situación que hemos descrito no responda más que a un espejismo provocado por los propios métodos empleados y sus resultados (Cabrera et al., 2004).

Otro de los aspectos interesantes que aporta este momento es la cronología del Auriñaciense arcaico y antiguo en esta región. Estratigráficamente, el Auriñaciense antiguo se superpone al Auriñaciense arcaico en toda la región estudiada (Arrizabalaga et al, en prensa); las dataciones $\mathrm{C}^{14}$ también corroboran este hecho, pero presentan una sincronía parcial de ambos tecnocomplejos en la región, no en el mismo yacimiento, pero sí entre varios. Esta problemática, que podría tener las mismas causas que hemos descrito en el párrafo anterior, se debe analizar también desde una perspectiva de ocupación del territorio y que, necesariamente, debe exceder el marco regional aqui tratado (Bon et al., 2002). Como ya hemos comentado, el Auriñaciense arcaico se asienta en la región vasco-cantábrica alrededor del 36.500 B.P. con unas características tecnotipológicas muy diferentes a las que va a presentar el Auriñaciense antiguo, cuyas primeras manifestaciones están en el yacimiento de Brassempouy con una datación para el nivel 2DE de 33.600 \pm 240 (Gif-11034). A partir de aquí volvemos a encontrar un solapamiento de dataciones que podrían implicar una coexistencia parcial de ambos tecnocomplejos. Esta coexistencia no se habría llevado a cabo en el mismo territorio, sino en regiones adyacentes, ya que no coinciden ambos tecnocomplejos en un mismo espacio (Bon et al., 2002).

\subsection{Paleoambiente}

El mayor volumen de información disponible sobre el paleoambiente durante la Transición entre el Paleolítico medio y superior, proviene de los estudios de Paleontología, Sedimentología y Paleobotánica. Estas analíticas se han aplicado sobre depósitos arqueológicos (generalmente en medios kársticos), si bien dentro de la Paleobotánica también se están incorporando los estudios palinológicos de depósitos de origen no antrópico. La información paleoambiental de este periodo corresponde a una sucesión de momentos puntuales, que en ningún caso permite establecer una visión continua, ni en su desarrollo cronológico, ni en la totalidad de los diferentes ambientes biogeográficos que caracterizan al área que nos ocupa. Diversos factores influyen en esta situación: escasez de depósitos con secuencias estratigráficas continuas; existencia de hiatos sedimentarios y/o polínicos; problemas de conservación de la materia orgánica; ausencia de depósitos limnéticos en determinadas zonas de la Cornisa Cantábrica y litoral atlántico; aplicación del principio del muestreo geológico en estudios paleopalinológicos; etc., y en ocasiones, la conjunción de varios de ellos limita aún más la información obtenida.

La variabilidad biogeográfica de este área, permite la existencia de una diversidad de zonas medioambientales que aportan una interesante variedad de recursos a la población humana, tanto a nivel de fauna, como de flora. La información referente a la fauna es mayor que la del mundo vegetal, pero ambas, así como la procedente de los estudios sedimentológicos reflejan el desarrollo de diversos episodios climáticos: El Castillo (Dari, 2003), Cueva Morín (Gónzalez Echegaray y Freeeman, 1971, 1978), Kurtzia (Muñoz et al., 1989/1990), Arrillor (Hoyos et al., 1999), Labeko Koba (Arrizabalaga y Altuna, 2000), Lezetxiki (Chaline 1970; Kornprobst y Rat, 1970; Altuna 1972; Sánchez-Goñi 1993), Ekain (Altuna y Merino, 1984), Gatzarria (Lévêque, Miskovsky, 1996), Isturitz (Bouchud 1951, 1952, Leroi Gourhan 1959), Duruthy (Arambourou, 1978), La Roche à Pierrot (Leroi-Gourhan, 1984; Leroyer, 1987), turbera de Biscaye (Mardones y Jalut, 1983). Durante este período, se evidencia la existencia de una sucesión de paisajes de ambientes fríos, caracterizados por una gran superioridad de los paisajes abiertos con un dominio de Poace- 
ae y Compositae junto a una mayor o menor representación de especies estépicas (en función del grado de humedad) en los que encontramos animales como Mammuthus primigenius, Coelodonta antiquitatis, Rangifer tarandus, etc. Estos ambientes, contrastan con los desarrollados durante los episodios de mejoría climática en los que la cubierta forestal aumenta presentando un predominio de taxones arbóreos mesófilos (Quercus, Corylus, Alnus, Betula, Ulmus, Tilia, Castanea, etc.) detectándose un incremento de la fauna asociada a las zonas boscosas. En líneas generales, parece ser que el final del Musteriense y el inicio del Paleolítico superior (Castelperroniense) coincide con los momentos finales del Interestadial Würmiense bajo unas condiciones climáticas benignas (Cueva Morín, Labeko Koba, La Roche-à-Pierrot, turbera de Biscaye), situación que evoluciona a un clima dominado por las condiciones adversas durante los períodos más antiguos del Auriñaciense. A este período correspondería, en el caso de Labeko Koba, la base del nivel Protoauriñaciense, mientras que al final del mismo, las condiciones ambientales tienden a mejorar, para volver a deteriorarse a lo largo del Auriñaciense antiguo.

Pese a este limitado volumen de información, las visiones puntuales que hasta el momento venimos recuperando son coherentes con la sucesión de fases de progresión y retroceso glaciar del Estadio Isotópico 3 de las secuencias isotópicas del hielo de Groenlandia (Dansgaard et al., 1993). Asimismo, los datos recuperados de secuencias limnéticas de las zonas litorales-sublitorales del sector noroeste del Cantábrico (GómezOrellana, 2002) indican un importante grado de inestabilidad ambiental que únicamente permitiría expansiones moderadas de la masa boscosa en aquellos territorios donde estuviesen acantonadas pequeñas poblaciones en áreas de refugio. Las características de este territorio han permitido la existencia de una interesante variedad de unidades biogeográficas a lo largo de todo el Cuaternario, circunstancia que no puede olvidarse al intentar aplicar un modelo unitario a la totalidad del mismo; por lo tanto, no procede cuestionar aquellas secuencias que no cumplen con aquél. La evolución ambiental a lo largo del Pleistoceno superior fue muy diversa, como lo tuvo que ser la respuesta que estas diferentes áreas biogeográficas dieron a la sucesión de periodos climáticos (Iriarte et al., 2005). De todos modos, en un futuro próximo, el volumen de información va a aumentar considerablemente debido a la incorporación de los estudios de nuevos depósitos (arqueológicos o no), así como a la revisión de algunos de los mencionados en este trabajo, como Lezetxiki y Cueva Morín.

\subsection{Economía cinegética}

El emplazamiento conocido de los yacimientos en el Musteriense y el Paleolítico superior inicial parecen reflejar la existencia de una cierta continuidad, localizándose a lo largo de la estrecha franja costera del mar Cantábrico, ocupan- do tanto la zona de bajas colinas de la Marina de Cantabria como los bordes de la cordillera cantábrica. La presencia de los yacimientos musterienses se sitúa mayoritariamente en los anchos valles de regiones montañosas de baja altitud (Cabrera-Valdés y Bernaldo de Quirós 1992) aunque la presencia de depósitos a lo largo de los ríos en los valles interiores, con zonas abruptas pero con altitudes entre 300 y 100 metros, son la evidencia que nos sugiere unas estrategias de subsistencia más especializada como la caza de la cabra (Baena et al., 2005).

Si comparamos la región cantábrica con otras zonas cercanas, como los Pirineos, observamos un primer aspecto fundamental. Geográficamente, la región cantábrica es un pasillo con una dirección de movilidad principal este-oeste, mientras que los Pirineos se encuentran situadas en zonas con posibilidades de expansión en otras direcciones. En el caso de los Pirineos, se detecta, junto a una dirección esteoeste, otra sur-norte (Bahn, 1984). Por un lado, encontramos una relación Mediterráneo-Atlántico junto a otra PirineosDordogne. En el caso cantábrico el eje fundamental es País Vasco-Asturias. La comunicación con el sur, tanto con el valle del Ebro, como con el valle del Duero, son posibilidades conocidas, pero por el momento no demasiado empleadas (Tarriño y Normand, 2002).

Como en los demás periodos del Paleolítico superior, los biotopos terrestres son los más utilizados, con alternancias entre ellos basados en la variedad de los tipos de yacimientos. El uso de recursos marinos es por el momento conocido, pero sin la intensidad que veremos en otros periodos como el Magdaleniense. Los principales restos de fauna relacionados con los problemas de transición en el cantábrico provienen de los yacimientos de Cueva Morín (Altuna, 1971, 1973), El Castillo (Dari, 2003), Ekain (Altuna, Mariezkurrena, 1984), Lezetxiki (Altuna, 1972) o Labeko Koba (Altuna, Mariezkurrena, 2000). Lo que nos interesa conocer en este momento es la presión cinegética de los grupos que ocuparon los diferentes yacimientos y su habilidad para obtener estos recursos clave. Los datos se muestran en la Tabla 1 para los principales ungulados del musteriense, y en la Tabla 2 para el Paleolítico superior inicial.

La comparación entre yacimientos muestra, en primer lugar, la existencia de claras diferencias en la cantidad de caza presente, así como la progresiva importancia del ciervo durante la Transición. El espectro de especies presentes en los yacimientos refleja la diversidad de hábitats accesibles desde los mismos y la disponibilidad de recursos para los grupos humanos que vivieron en el área. También es útil remarcar la representación de carnívoros o carroñeros, que nos puede dar una indicación de la intensidad de la ocupación.

Los resultados del estudio estacional de las cuevas de El Castillo y Cueva Morín pueden verse a la luz de la caracterización de los yacimientos. Éstos se sitúan, a pocos kilómetros de los recursos de la costa, ríos, valles, bosques y montañas. 
Ninguna de las cuevas muestra diferencias dramáticas entre prácticas subsistentes en el Musteriense y el Paleolítico superior inicial, ni tampoco en cuanto al uso del yacimiento en términos de caza estacional y edad de los animales de presa individuales. Sin embargo, cada yacimiento revela un patrón propio que refleja elecciones de comportamiento por parte de los ocupantes musterienses y del Paleolítico Superior inicial. Los resultados de los análisis de crecimiento de los dientes realizados (Pike-Tay et al., 1999) sugieren unas capturas anuales a lo largo del Musteriense y del Auriñaciense de Transición de El Castillo. A pesar de esto, los patrones estacionales de la muestra musteriense difieren en algo de la muestra de la unidad superior. La mayoría de los animales fueron cazados desde finales de otoño hasta la primavera o comienzos del verano en los niveles musterienses y del invierno hasta la primavera en el Auriñaciense de Transición. En Cueva Morín, los resultados de estos análisis sugieren que los animales se cazaron durante el final del otoño y/o el invierno durante el Musteriense y el Auriñaciense arcaico. Los cambios en los patrones estacionales de caza y en la duración y naturaleza de las ocupaciones de estos yacimientos indudablemente reflejan cambios en los patrones de uso de la tierra, innovaciones tecnológicas y estrategias de recolección de recursos que a su vez son una respuesta a las fluctuaciones climáticas y a cambios en las dinámicas de población regional.

Considerando las cantidades de animales representados en cada yacimiento y relacionándola con la cantidad de carne que estos aportan, podemos comprobar (Bernaldo de Quirós 1982) que ésta representa una cantidad limitada, observando una cierta distinción entre niveles, pocos con mucha cantidad y muchos con poca. Esto nos permite proponer una cierta dicotomía entre los yacimientos, con algunos de ellos utilizados por la generalidad del grupo, y otros serán ocupados por un segmento del mismo. Esta alternancia también presenta algunos yacimientos con caza especializada, con ejemplos como El Conde o El Esquilleu, especializados en la caza de cápridos (Bernaldo de Quiros, 1982; Baena et al., 2005). Yacimientos con caza especializada en ciervos se detectan también en este momento, como en la cueva de El Castillo (Cabrera-Valdés, 1984; Dari, 2003), con una importancia numérica que ha sido confirmada en las recientes excavaciones. Se identifican yacimientos de agregación, generalmente grandes en superficie, ricos en industria y restos de fauna (generalmente de ciervo) como El Castillo, junto a otros de disgregación, ocupados por algunos individuos, con menos variabilidad tipológica y en los que los restos de fauna presentan cantidades equilibradas de ciervo y otros animales. En esta categoría podriamos incluir Cueva Morín, Lezetxiki, Labeko Koba o El Otero, junto a ocupaciones muy efímeras (como podría ser el caso de Ekain). El tercer grupo es el ya citado de los yacimientos especializados en la caza de cabras como Esquilleu o el Conde. Así, vemos como los ya- cimientos se sitúan en un espacio en el que observamos una gestión inteligente de los recursos tanto durante el Musteriense como en el Paleolítico Superior Inicial, lo que aporta un elemento nuevo en la discusión de la "Transición".

\subsection{Tecnología lítica}

El estudio de los esquemas operativos de los diferentes conjuntos arqueológicos aquí tratados nos permite dar una visión más completa que la proporcionada por los estudios tipológicos clásicos, ya que permite reconocer diferentes tradiciones entre los diferentes conjuntos industriales involucrados en la Transición entre el final del Musteriense y los inicios del Paleolítico superior en la región Cantábrica. Estas diferencias "tradicionales" no sólo se identifican entre las dos facies tratadas en este trabajo, sino también dentro de las mismas (Arrizabalaga et al., en prensa; Bon, 2002; Bon et al., 2006).

\subsubsection{Final del Musteriense}

Para abordar la caracterización tecnológica del final del Musteriense contamos con el estudio de los niveles 11 y 12 de Cueva Morín (Maíllo Fernández, 2003), el nivel 20e de la cueva de El Castillo (Sánchez-Fernández, 2005), el estudio realizado en la cueva del Esquilleu (Baena et al., 2005, 2006) y los datos preliminares de Axlor (Ríos, 2005; González Urquijo et alli, 2006) y Covalejos (Martín et al., 2006). Los esquemas operativos presentan una gran variabilidad. Así, en la cueva del Esquilleu (situada en la zona de Picos de Europa), la variabilidad interna es bastante variada en los diferentes niveles: nivel XI tipo Quina, nivel IX tipo Levallois recurrente unipolar y en el nivel III el esquema operativo principal es de tipo discoide jerarquizado. Esta variabilidad también la observamos en el yacimiento de Axlor (Ríos, 2005; González Urquijo, et al., 2006) ya que en el nivel $\mathrm{N}$ presenta un esquema operativo principal de tipo Levallois orientado a la obtención de lascas y puntas; mientras que los niveles $B, C$ y $D$ presentan un esquema operativo principal de tipo Quina, y cuya finalidad es la obtención de lascas espesas con dorso, destinadas a la elaboración de raederas con retoque Quina.

En la zona central de Cantabria, el esquema operativo principal es de concepción discoide en los yacimientos de $\mathrm{El}$ Castillo (nivel 20e) y Cueva Morín (niveles 11 y 12) o La Flecha (Castanedo, 2001). Se emplean dos métodos: uno unifacial con las superficies jerarquizadas y otro, bifacial, en el que no existe tal jerarquización. Este último, en Cueva Morín, se emplea con materias primas de grano grueso como la ofita y la arenisca. Estas materias primas, al aparecer en nódulos de mayor tamaño, permiten la obtención de soportes más largos y espesos (Maíllo Fernández, 2003).

En el Castillo existe un esquema operativo destinado a la elaboración de hendedores. Para ello, se emplean materias primas de grano grueso y cuyas dimensiones permiten la ela- 
boración de este tipo de instrumentos, como son la arenisca y la ofita (Sánchez-Fernández, 2005). En los niveles 11 y 12 de Cueva Morín, no se encuentran hendedores, pero sí en los seis niveles infrayacentes descritos como Musterienses.

Uno de los esquemas operativos más interesante de los que han sido identificados en el Musteriense final cantábrico es el de la producción de hojitas (Cabrera et al., 2000; Maillo Fernández, 2001; Maíllo Fernández et al., 2004; Sánchez-Fernández \&t Maillo-Fernández, 2006). Estas hojitas se consiguen a partir de núcleos de morfología prismática y gestión unipolar semienvolvente. Dos variantes son posibles: una sola tabla o dos tablas adyacentes. En el primero de ellos, la explotación se gestiona a partir de soportes frontales y soportes obtenidos en la confluencia entre la tabla y el flanco. Éstos últimos, mantienen el cintrado del núcleo. En los núcleos con dos tablas adyacentes, la arista entre ambas mantiene al cintrado del mismo. Los soportes, aunque escasos, están retocados, tanto en Cueva Morín (una hojita de dorso y una hojita con retoque semiabrupto) como en El Castillo (dos hojitas con retoque semiabrupto alterno tipo Dufour) y algunas de ellas presentan huellas de uso y restos de posible enmangue (Gutiérrez, 2006). También, en el vecino yacimiento de Covalejos (nivel H), encontramos un esquema operativo de hojitas a partir de la captura oportunista de aristas de productos de talla desechados (Martín et al, 2006). Desde el sistema de análisis analítico y estructural, constatamos también este proceso de laminaridad en conjuntos como Lezetxiki IV (Arrizabalaga, 1995), Arrillor (Hoyos et al., 1999) y en los yacimientos del vecino País Vasco francés de Abri Olha 2 y Gatzarria (Laplace y Sáenz de Buruaga, 2000; 20022003).

\subsubsection{Auriñaciense de Transición}

Tecnocomplejo identificado, por el momento, en los niveles $18 \mathrm{~b}$ y $18 \mathrm{c}$ de la cueva de El Castillo (Lloret y Maíllo-Fernández, 2006). Tecnológicamente, la producción lítica está dominada por los esquemas operativos de concepción discoide con dos métodos bien definidos: unifacial y bifacial. El desarrollo del débitage es similar al descrito en el final del Musteriense.

Se ha identificado, de forma más discreta, un esquema operativo laminar de hojitas muy similar al ya descrito para el final del Musteriense. La mayoría de los núcleos están realizados sobre cuarcita, siendo muy escasos los de sílex. La fase de mise en forme es sencilla, existiendo una adecuación morfológica del núcleo en relación con los soportes deseados. No se aprecian negativos en los núcleos que puedan advertir de la existencia de crestas anteriores ni postero-laterales. El inicio del débitage se lleva a cabo mediante la extracción de una lasca de entame. A partir de este tipo de piezas, la tabla se abre hacia los flancos mediante la extracción de soportes de morfología tendente a laminar que presentan un lateral cortical.
Los núcleos presentan una morfología prismática y una explotación unipolar. Los soportes obtenidos mediante este tipo de explotación correspondería a hojitas o lasquitas laminares de no demasiada longitud y relativamente anchas.

Aunque consideramos que el esquema de morfología prismática y gestión unipolar es el principal en estos dos niveles de la cueva de El Castillo, constatamos también otros esquemas operativos de tipo raspador-carenado y buril-carenado.

\subsubsection{Chatelperroniense}

Este tecnocomplejo tiene una presencia menor en la región cantábrica. Tras su descubrimiento en Cueva Morín (González Echegaray y Freeman, 1971), ha sido identificado en unos pocos yacimientos en los que, a excepción de esta cueva y Labeko Koba en el País Vasco (Arrizabalaga \& Altuna, 2000), los vestigios arqueológicos son escasos o dudosos (Maillo-Fernández, 2007).

Tras los estudios tecnológicos realizados recientemente en Labeko Koba (Arrizabalaga, 1995; 2000b) y Cueva Morín (Maíllo Fernández, 2003) podemos afirmar que el esquema operativo más numeroso es de concepción discoide, bajo el método unifacial con superficies jerarquizadas. Dicha explotación se realiza sobre todas las materias primas.

Desde un punto de vista cualitativo, los esquemas operativos laminares resultan más interesantes y relevantes. Dos son los métodos empleados: prismático unipolar y prismático bipolar. El primero de ellos se realiza a partir de nódulos de morfología cúbica en la que la preferencia para el inicio de explotación es la cresta de núcleo. A partir de aquí, la tabla se abre hacia uno de los flancos, que son perpendiculares a ésta. Los soportes obtenidos son hojas rectilíneas, no muy espesas y con ligera curvatura distal. El plano de percusión es liso y se produce mediante la extracción de una lasca que prepara la superficie. Ésta se reaviva durante el desarrollo del débitage.

Por su parte, el método prismático bipolar mantiene los mismos requisitos morfológicos que el anterior, pero con dos planos de percusión que son preparados de manera idéntica al anterior método. A partir de éste se obtienen hojas de morfología rectilínea y que son destinadas, en su gran mayoría a la elaboración de puntas de Chatelperrón. Se ha constatado en algunos yacimientos chatelperronienses como Roc-deCombe (Pelegrin, 1995) como uno de las direcciones de extracción era la principal, desde donde se obtenían las hojas destinadas a confeccionar puntas de Chatelperrón, mientras que los soportes extraídos desde el sentido opuesto tenían como función acondicionar el cintrado de la tabla. En los niveles chatelperronienses cantábricos no hemos podido discenir esta modalidad de explotación.

En el nivel Chatelperroniense de Cueva Morín no existe una producción específica de hojitas ya que las que aparecen en dicho nivel son obtenidas de manera accidental durante la explotación de hojas. 


\subsubsection{Protoauriñaciense/Auriñaciense arcaico}

Los yacimientos que permiten una identificación tecnológica de este tecnocomplejo son: Cueva Morín (niveles 8 y 9); El Castillo (nivel 16) y Labeko Koba (nivel VII), a partir de los cuales podemos identificar los siguientes aspectos en cuanto a la producción lítica (Arrizabalaga, 1995; 2000b; Maíllo-Fernández 2003, 2006; Cabrera et al., 2002):

a) Existe un continuum entre la producción de hojas y de hojitas.

b) El método de explotación más empleado es el que se realiza a partir de núcleos prismáticos por medio de una gestión unipolar. El inicio del débitage se realiza a partir de piezas de entame.

c) Dos tipos de soportes son obtenidos: hojas y hojitas de perfil rectilíneo que presentan una buena regularidad y que serían obtenidas en el centro de la tabla; las hojas y hojitas de perfil más curvo, sobretodo en la parte distal con cortex lateral (que puede ser sustituido por neocrestas), serían obtenidas en la intersección entre la tabla y el flanco. Estas extracciones tienen como objetivo controlar el cintrado de la tabla y permite obtener las condiciones volumétricas del núcleo para generar el primer tipo de soportes.

d) Las hojitas son empleadas para realizar hojitas $D u$ four, subtipo Dufour (Demars y Laurent, 1992).

e) En algunos yacimientos (Gatzarria y Cueva Morín) destacan el débitage de lascas bajo esquemas de concepción discoide, que tiene su reflejo entre el material retocado, donde resultan muy abundantes las piezas de fondo común.

\subsubsection{Auriñaciense antiguo:}

Existe un mayor número de niveles clasificados como Auriñaciense antiguo en la zona de estudio. Aunque aquí trataremos los más significativos: Cueva Morín (niveles 7 y 6 ) y los niveles VII, VI y V de Labeko Koba (Arrizabalaga, 1995, 2000; Cabrera et al., 2004).

a) El rasgo tecnológico más relevante es que existe una disociación entre la producción de hojas y la de hojitas. La primera se efectúa a partir de núcleos prismáticos de gestión unipolar, mientras que la segunda se lleva a cabo mediante esquemas operativos de tipo raspador carenado.

b) La producción de hojas presenta una sistematización destacable en todos los yacimientos. La morfología prismática y la gestión unipolar serían los elementos más importantes, pero las convergencias se observan mejor en otros ámbitos más específicos como los soportes robustos y con cierta curvatura, neocrestas en las modalidades de reavivado o el inicio del débitage a partir de piezas de entame.

c) La producción de hojitas se presenta más homogénea en cuanto a métodos y modalidades empleadas para llevarla a cabo. Ésta se realiza a partir de núcleos carenados cuya tabla es, generalmente de morfología triangular.

\subsection{Tipología}

En este apartado trataremos de trazar las principales directrices que guian la confección y empleo de diferentes artefactos líticos u óseos durante este periodo convulso. Vaya por delante la observación de que, si somos capaces de aplicar un mismo protocolo metodológico al estudio de las colecciones del período transicional resultará terriblemente complicado establecer criterios frontera, que nos separen de modo nítido las situaciones de final del Musteriense y del inicio del Paleolítico superior. En este punto del texto ya han sido mencionados ampliamente la gestión de las materias primas líticas y la tecnología. Si estudiamos los extremos de un espectro cronológico que arranque hacia el 42.000 y concluya en el 27.000 nos resulta sencillo observar fuertes contrastes entre ambas situaciones. Sin embargo, si intentamos observar cuándo y de qué modo se van introduciendo estos cambios, resultará complicado establecer valores inamovibles y que coincidan en el tiempo, dentro además de un amplio rango geográfico.

Caracteriza al Musteriense una industria ósea poco elaborada, en la que retocadores, algunos punzones y útiles "tallados" sobre hueso rememorando técnicas líticas constituyen el principal componente de las series. No puede considerarse (con alguna notable excepción) que la adición de azagayas desde el Chatelperroniense-Protoauriñaciense introduzca una notable variación en estos conjuntos, toda vez que representan casi siempre elementos de poco peso cuantitativo en las series: éstas continúan aún, hasta avanzado el Gravetiense, manteniendo unos apoyos comunes en los tipos señalados. A partir del Auriñaciense antiguo (el Chatelperroniense en Labeko Koba, el Protoauriñaciense en el caso de Isturitz) van a irse introduciendo de modo puntual las azagayas aplanadas (algunas de ellas, auténticas azagayas de base hendida), que aumentarán su peso cuantitativo a medida que redondean su sección.

De modo más matizado, podríamos establecer similares consideraciones para la industria lítica. La progresión del sílex como materia prima de referencia, el proceso de Leptolitización (laminarización y microlitización), la progresiva implantación de técnicas de debitado laminares y microlaminares o la sustitución de los grupos tipológicos de sustrato (raederas y denticulados) por otros que vienen siendo vinculados al Paleolítico superior (buriles, raspadores, elementos de dorso o perforadores, entre otros) son claramente visibles en los extremos del lapso de 15.000 años, arriba indicado. Sin embargo, deben ser valorados en términos cuantitativos siempre, puesto que ninguno de ellos marca una línea absolutamente discriminante que nos permita atribuir una colección, sin dudas, a alguno de los tecnocomple- 
jos citados. Como quiera que las tipologías al uso han empleado una lista diferente según la presunción a priori del carácter musteriense o auriñaciense del conjunto, esta anomalía metodológica ha generado una gravísima distorsión en la percepción de la transición. La aplicación de un único criterio metodológico al conjunto de las series, a partir de la Tipología Analítica, permite estimar en términos cuantitativos la evolución diacrónica de estas variables.

Restringiéndonos a la valoración tecnotipológica de las series señalaremos que hoy por hoy resulta más indicado referirnos al final del Musteriense, que al Musteriense final. No ha sido descrita ninguna caracteristica industrial en el registro lítico (el más abundante) que nos permita romper con el carácter ajerárquico desde la perspectiva cronológica del Musteriense: no podemos distinguir, sólo por su industria lítica, un Musteriense final. Dentro del Musteriense de la región descrita nos encontramos con una abundancia muy significativa (en ocasiones superior al $80 \%$ de la serie) de los elementos de sustrato, entre los que destacan siempre las raederas. Estos conjuntos se hayan enriquecidos por porcentajes menores, aunque variables, de componentes supuestamente "leptolíticos", tales como buriles, écaillés, raspadores, perforadores o piezas de dorso, junto a otros sobre soporte masivo y supuestamente arcaizantes. Será el ascenso progresivo de los componentes leptolíticos de la industria el que determinará, en último término, que la apariencia musteroide de un determinado conjunto vaya abriendo espacio a la apariencia auriñacoide.

En este proceso, relativamente pausado y continuo en el registro tipológico, el Chatelperroniense $u$ otras formas descritas como transicionales en el medio cantábrico (El Conde, El Castillo, Lezetxiki) pueden tener una apariencia más o menos próxima al mundo musteriense, en la que se insertan componentes leptolíticos como las puntas de chatelperron, buriles o porcentajes variables de soportes laminares con retoque simple lateral o bilateral.

La serie lítica indiscutiblemente leptolítica se inicia con el Protoauriñaciense, complejo caracterizado por la presencia masiva de laminitas de retoque semiabrupto (algunas, características Dufour) y un consiguiente repunte de los modos de retoque abrupto - primero- y buril -posteriormente-. Este desarrollo es bien conocido y como resultado del mismo, al concluir el Auriñaciense evolucionado y dar éste paso al Gravetiense, la presencia de elementos líticos de sustrato resulta bastante marginal.

\section{RECAPITULACIÓN Y PERSPECTIVAS DE LA INVESTIGACIÓN}

El cambio de perspectiva que se ha producido durante los últimos quince años para el conocimiento de la Transición entre el Paleolítico medio y superior ha sido muy notable, al menos en la región aquí descrita. Se ha incrementado notablemente el ritmo de los trabajos de campo, se ha estandarizado la metodología de estudio, al tiempo que se mejoraba su resolución y se han vuelto a cuestionar los tópicos más extendidos sobre este periodo tan debatido. Como no podía ser de otro modo, tantas novedades acumuladas originan un estado de opinión diferente al que creíamos tener a principio de los años noventa.

Cada una de las temáticas tratadas en el segundo apartado, y algunas no tratadas, ha dado lugar a cierta discusión. Transversalmente a ellas (a cuestiones como la cronología precisa de la Transición, el surgimiento del comportamiento gráfico o la caracterización antropológica de los autores de los tecnocomplejos del periodo) se entrelazan problemas más complejos: en la llamada Transición, ¿predomina la continuidad o la ruptura?; ¿existe un único diseño de Transición o cabe aceptar que en diferentes situaciones se han registrado diversas respuestas?; ¿cuál es la relevancia de la caracterización filética de los protagonistas del registro (neandertales o cromañones) en el proceso descrito? y ¿cuál la de las diferentes aproximaciones metodológicas y epistemológicas con la que los distintos prehistoriadores abordan el problema? Finalmente, ¿se produjo efectivamente una situación de convivencia en el espacio y el tiempo de neandertales y cromañones o esta imagen es sólo una recreación literaria que nace de un espejismo provocado por la baja resolución del registro paleolítico?

Entendemos en este artículo que no se pueden responder directamente estas preguntas trascendentes sin atender primero aquellas más cercanas que han sido planteadas en primer término. El principal motivo de la crisis epistemológica que afecta al estudio del primer Paleolítico superior desde 1980 radica probablemente en haber dado por supuestos (y comprendidos) demasiados marcos generales y clasificaciones universales. Siendo cierto que es preciso encontrar un equilibrio entre la comprensión de los procesos a escala universal y local, opinamos que la renovación del conocimiento de este periodo pasa aún por la ampliación de la base documental (dataciones, analíticas, trabajos de campo, revisión de secuencias antiguamente excavadas y publicación de todas estas informaciones). Nosotros mismos nos percatamos de que entre los yacimientos presentados en este texto, sólo uno ha sido descubierto y excavado por primera vez en las dos últimas décadas. La diversidad geográfica de la ubicación de estos lugares (diferencias en el acceso a los recursos, en el ecosistema en que se insertan, en las condiciones de habitabilidad) no ha sido aún suficientemente valorada, salvo, quizás, en los estudios paleoambientales. Este conjunto de informaciones debe ser adecuadamente contextualizado en un marco regional laxo, sin fronteras artificiales, en el que creemos que no es correcto hacer converger a los datos, si estos muestran (como suele ser el caso) algunas diferencias notables. 
En este sentido, puede ser paradigmático el ejemplo de la cronología absoluta (siempre en relación con la adscripción tecnocultural de cada conjunto): sobre una base aún estrecha de informaciones, distribuidas (por periodos, por áreas, por yacimientos, por la época en la que se efectuaron las dataciones, por método de obtención, por soporte empleado, etc.) de modo muy asimétrico, no puede llamar la atención que los mismos niveles de Cueva Morín, datados con cuarenta años de distancia, haya una dife- rencia significativa en el promedio de los resultados. Si queremos avanzar en el conocimiento de este periodo debemos ser capaces de aceptar e interpretar la diferencia, antes de proponer procesos y modelos de validez universal. Siendo necesario abordar aquel nivel de abstracción, quizás sea un poco prematuro hacerlo todavía, y en todo caso no vemos modo alguno de presentar un modelo "cerrado" y casi definitivo para interpretar un periodo de cierta complejidad.

\section{BIBLIOGRAFIA}

AltunA, J. (1971): Los mamíferos del yacimiento prehistórico de Morín (Santander), in González Echegaray \&t Freeman, eds., Cueva Morín. Excavaciones 1966-1968, 369-400.

AltunA, J. (1972): Fauna de Mamiferos de los yacimientos prehistóricos de Guipúzcoa, Munibe, 22 (3/4), 165-168.

AltunA, J. (1973): Fauna de mamíferos de la Cueva de Morin (Santander), in González Echegaray \& Freeman, eds., Cueva Morín. Excavaciones 1969, 281-292.

AltunA, J. y Merino, J. M. -dir- (1984): El yacimiento prehistórico de la cueva de Ekain (Deba, Gipuzkoa), Colección Barandiarán, Sociedad de Estudios Vascos, San Sebastián.

AltunA, J. Y MARIEZKURRENA, K. (1984): Bases de subsistencia de origen animal en el yacimiento de Ekain, in Altuna y Merino, eds., El yacimiento prehistórico de la cueva de Ekain (Deba, Guipúzcoa), 211-280.

Altuna, J.: Baldeón, A.; MariezKurRenA, K. -eds- (1990): La cueva de Amalda (Zestoa, País Vasco). Ocupaciones paleolíticas y postpaleoliticas, Colección Barandiarán, 4, Sociedad de Estudios Vascos, San Sebastián.

Altuna, J.; Armendariz, A.; Etxebarria, F.; Mariezkurrena, K.; Peñalver, X. y ZUMALABE, F. J. (1995): Carta arqueológica de Guipuzkua, vol II (cuevas), Munibe, suplemento 10.

AltunA, J. y MARIEZKURRENA, K. (2000): Macromamíferos del yacimiento de Labeko Koba (Arrasate, País Vasco), in Arrizabalaga y Altuna, eds., Labeko Koba (País Vasco). Hienas y humanos en los albores del Paleolítico superior, 107-181.

Arambourou, R. (1978): Le gisément préhistorique de Duruthy, à Sorde L'Abbaye (Landes), Bilan des Recherches de 1958 a 1975, Mémoires de la Société Préhistorique Française, 13, París.

Arrizabalaga, A., 1995: La industria litica del Paleolítico Superior Inicial en el Oriente Cantábrico. Ph.D. dissertation. Universidad del País vasco.

Arrizabalaga, A., 2000a: El yacimiento arqueológico de Labeko Koba (Arrasate, País Vasco). Entorno. Crónica de las investigaciones. Estratigrafia y estructuras. Cronología absoluta. In Labeko Koba (Pais Vasco). Hienas y Humanos en los albores del Paleolítico superior, edited by A. Arrizabalaga and J. Altuna, pp. 15-72. Munibe, 52, San Sebastián.

Arrizabalaga, A., 2000b: Los tecnocomplejos líticos del yacimiento arqueológico de Labeko Koba (Arrasate, País Vasco). In: Arrizabalaga et Altuna (éds.), Labeko Koba (País Vasco). Hienas y Humanos en los albores del Paleolítico superior. Munibe 52, 193-343. Aranzadi, San Sebastián.

Arrizabalaga, A. (2006): Lezetxiki (Arrasate, Pais Vasco). Nuevas preguntas acerca de un antiguo yacimiento. En En el centenario de la cueva de El Castillo: el ocaso de los Neandertales, eds. V. CabreraValdés, F. Bernaldo de Quirós y J. M. Maillo-Fernández,. Santander: UNED-CajaCantabria, 291-309.

Arrizabalaga, A. y Altuna, J. -eds- (2000): Labeko Koba (País Vasco). Hienas y Humanos en los albores del Paleolítico superior, Munibe (antropología-Arkeologia), 52.
Arrizabalaga, A.; Bon, F; Maillo-Fernández, J.M., Normand, Ch. y Ortega, I. (en prensa): Territoires et frontières de l'Aurignacien dans les Pyrénées occidentales et les Cantabres. En Cazals, N. (ed): Frontières naturelles et frontières culturelles dans les Pyrénées préhistoriques, Actes de la table.ronde de Tarascon-sur-Ariege, 2004.

Baena, J.; Carrión, E.; Ruiz, B.; Ellwood, B.; Sese, C.; Yravedra, J.; JordÁ, J.; Uzquiano, P.; VelázQuez, R.; Manzano, I.; Sánchez-Marco, A. y HerNÁNDEZ, F., 2005: Paleoecología y comportamiento humano durante el Pleistoceno Superior en la comarca de Liébana: la secuencia de la cueva de El Esquilleu (Occidente de Cantabria, España), in Neandertales cantábricos. Estado de la cuestión, eds. R Montes y J. A. Lasheras. (Monografias del Museo de Altamira 20) Santander: Museo de Altamira, 461-87.

BAenA, J.; CARRIÓN, E. y VelÁZQuez, R., 2006. Tradición y coyuntura: claves sobre la variabilidad del musteriense occidental a partir de la cueva del Esquilleu, En En el centenario de la cueva de El Castillo: el ocaso de los Neandertales, eds. V. Cabrera-Valdés, F. Bernaldo de Quirós y J. M. Maíllo-Fernández,. Santander: UNED-CajaCantabria, 249-67.

BAHN, P. G., 1984: Pyrenean Prehistory. A paleoeconomic survey of the French sites, pp. 510. Aris y Philips. Warmister.

Bernaldo de Quirós, F. 1982: Los Inicios del Paleolítico Superior. Centro de Investigación y Museo de Altamira, 8, Dirección General de BeIlas Artes y Archivos Madrid.

Bon, F., 2002a, L'aurignacien entre Mer et Océan. Réflexion sur l'unité des phases anciennes de l'Aurignacien dans le sud de la France. Société Préhistorique Française, Mémoire XXIX. Paris.

Bon, F.; Maillo-FernándeZ, J. M. y ORTEGA, D. (2006): El Auriñaciense arcaico peninsular y sus relaciones con el sur de Francia: una aproximación tecnológica. En En el centenario de la cueva de El Castillo: el ocaso de los Neandertales, eds. V. Cabrera-Valdés, F. Bernaldo de Quirós y J. M. Maillo-Fernández, Santander: UNED-CajaCantabria, 325-345.

Bouchoud, J. (1951): Etude paléontologique de la faune d'Isturitz, Mammalia, 15, 184-203

Bouchoud, J. (1952): Les oiseaux d'Isturitz, Bulletin de la Société Préhistorique Française, 49, 450-459.

CABRERA VALdÉS, V., 1983. Notas sobre el Musteriense cantábrico: el "Vasconiense". In Homenaje al Prof. Martín Almagro Basch, ed M. Fernández-Miranda, Ministerio de Cultura, 131-141.

Cabrera-Valdés, V., 1984, El yacimiento de la Cueva de El Castillo. (Puente Viesgo, Santander). C.S.I.C. Madrid.

CABRERA-VALdÉS, V. y BISCHOFF, J. (1989): Accelerator 14C ages for basal Aurignacien at El Castillo Cave. Journal of Archaeological Science 16: 577-584.

Cabrera-Valdés, V. y Bernaldo de Quirós, F. (1992): Approaches to the Middle Palaeolithic in Nothern Spain. En Dibble. H. y Mellars, P. A. Middle Paleolithic adaptation: behavior and variability. Monographs, 72. University of Pennylvania: 97-112.

Cabrera-Valdés, V.; Lloret Martínez de la Riva, M. y Bernaldo de Quirós, F. 1996a: Materias primas y formas líticas del Auriñaciense Arcaico de la Cueva del Castillo Puente Viesgo, Cantabria. Espacio, Tiempo y Forma, Serie I. 9:141-158 
Cabrera-Valdés, V.; Valladas, H.; Bernaldo de Quirós, F. y Hoyos, M., 1996b: La Transition Paléolithique moyen-Paléolithique supérieur à El Castillo (Cantabrie): nouvelles datations par le carbone-14. C.R. de la Academie des Sciences 322 (série II), 1093-8.

Cabrera-Valdés, V. y Bernaldo de Quirós, F. (2000): Excavaciones arqueológicas en la cueva de El Castillo (Puente Viesgo), 1980-1999. En Actuaciones arqueológicas en Cantabria, 1984-1999. Gobierno de Cantabria: 23-34.

Cabrera-Valdés, V.; Pike-Tay, A.; Lloret, M. y Bernaldo de Quirós, F. (2000): Continuity patterns in the Middle-Upper Palaeolithic Transition in Cantabrian Spain. En Stringer, C.B.; Barton, R.N.E. y Finlayson, J. C. (eds): Neandrethals on the edge. Oxbow Books: 85-93.

Cabrera-Valdés, V.; Maíllo-Fernández, J. M. y Bernaldo de Quirós, F., 2000, Esquemas operativos laminares en el Musteriense final de la cueva del Castillo (Puente Viesgo, Cantabria). Espacio, Tiempo y Forma, Serie / 13, 51-78.

Cabrera-Valdés, V.; Maíllo-Fernández, J. M.; Lloret, M y Bernaldo De QuiRós, F., 2001: La transition vers le paléolithique supérieur dans la grotte du Castillo (Cantabrie, Espagne): la couche 18. L'Anthropologie 105: 505-532.

Cabrera-Valdés, V.; Bernaldo de Quirós, F.; Maíllo-Fernández, J. M.; VaLLADAS, H. Y LLORET, M., 2002: El Auriñaciense arcaico de El Castillo (Cantabria): descripción tecnológica y objetivos de la producción. In Autour des concepts de Protoaurignacien, d'Aurignacien archaïque, initial et ancien, edited by F. Bon, J. M. Maillo-Fernández and D. Ortega, pp. 67-86. Espacio, Tiempo y Forma 15, Madrid.

Cabrera-Valdés, V.; Arrizabalaga, A.; Bernaldo de Quirós, F. y Maillo-FerNÁNDEZ, J. M. (2004): La Transición al Paleolítico superior y la evolución de los contextos Auriñacienses (50.000-27.000 B.P.). En Fano, M. (ed): Las sociedades del Paleolitico en la región cantábrica. Kobie, Anejo 8: 141-208.

Cabrera-Valdés, V.; Bernaldo de Quirós, F.; Maillo-Fernández, J. M.; LloRET, M.; TeJeRo, J. M. y MORÁn, N., 2005: La Unidad 18 de la cueva de El Castillo (Puente Viesgo, Cantabria): el Auriñaciense de Transición. Definición e implicaciones. Sautuola XI: 11-35.

Cabrera-Valdés, V.; Bernaldo de Quirós, F.; Maillo-Fernández, J. M.; PikeTAY, A. y GARRALDA, Ma. D. (2005): Excavaciones en El Castillo: veinte años de reflexiones. En R Montes y J. A. Lasheras (eds). Neandertales cantábricos. Estado de la cuestión (Monografias del Museo de Altamira 20) Santander: Museo de Altamira: 505-526.

CARBAllo, J., 1923: Excavaciones en la Cueva del Rey en Villanueva (Santander). Madrid: Junta Superior de Excavaciones y Antigüedades.

CASTANEDO, I., 2001: Adquisición y aprovechamiento de los recursos líticos en la Cueva de La Flecha (Cantabria). San Sebastián, Munibe 53, 3-18.

Chaline, J. (1970): Pliomys lenki, forme relique dans la microfaune du Würm ansien de la grotte de Lezetxiki (Guipúzcoa, Espagne), Munibe, 22 (1/2), 43-49

Dansgaard, W.; Johnsen, S. J.; Clausen, H. B.; Dahl-Jensen, D.; Gundestrup, N.S.; HAMmeR, C. U.; HVIDBERG, C. S.; StefFenSEN, J. P.; SVERnBJÖRNSDOTtIR A. E.; JOUZEL, J. Y BOND, G. (1993): Evidence for general instability of past climate form a 250-kyr ice-core record, Nature 364, 218-220.

DARI, A., 2003: Comportaments de subsistance pendant la transition Paléolithique moyen-Paléolithique supérieur en Cantabrie à partir de l'étude archéozoologique des restes osseux des gends mammiferes de la grotte d'El Castillo (Puente Viesgo, Espagne). Tesis doctoral. Museum National d'Histoire Naturelle. Paris

Demars, P-Y. y LAURENT, P. (1989): Types d'outils lithiques du Paléolithique supérieur en Europe. CNRS, p.177.

FORTEA, J., 1995: Abrigo de la Viña. Informe y primera valoración de las campañas 1991-1994. Excavaciones Arqueológicas en Asturias, 1991-94: 19-32.

Gómez-OrellanA, L. (2002): El último ciclo Glaciar-Interglaciar en el litoral del NW Ibérico: dinámica climática y paisajistica. Tesis Doctoral. Escola Politéctnica Superior. Universidade de Santiago de Compostela.
GónZalez-Echegaray, J. y Freeman, L. G. (1971): Cueva Morín: excavaciones 1966-1968, Publicaciones del Patronato de las cuevas prehistóricas de la provincia de Santander, 6.

GonzÁlez-Echegaray, J. y L. G. Freeman (eds.), 1973. Cueva Morín. Santander: Publicaciones del Patronato de las cuevas prehistóricas de la provincia de Santander, $X$

GónZalez-Echegaray, J. y Freeman, L. G. (1978): Vida y muerte en Cueva Morín, Institución Cultural de Cantabria, Colección de Bolsillo, 7 Santander.

González URQuiJo, J. E.; IBÁÑEZ, J. J.; Ríos, J. y BouRguignon, L., 2006: Aportes de las nuevas excavaciones en Axlor sobre el final del Paleolítico Medio, in En el centenario de la cueva de El Castillo: el ocaso de los Neandertales, eds. V. Cabrera-Valdés, F. Bernaldo de Quirós y J. M. Maillo-Fernández,. Santander: UNED-CajaCantabria, 269289.

GutiérREZ, C. (2006): Los niveles 16 a 21 de la cueva de El Castillo. Las huellas de uso. in En el centenario de la cueva de El Castillo: el ocaso de los Neandertales, eds. V. Cabrera-Valdés, F. Bernaldo de Quirós y J. M. Maillo-Fernández. Santander: UNED-CajaCantabria, 513-520.

Hoyos, M.; SÁenz de Buruaga, A. y Ormazabal, A. (1999): Cronoestratigrafía y paleoclimatología de los depósitos prehistóricos de la cueva de Arrillor (Araba, País Vasco), Munibe (Antropologia-Arkeologia) 51, 137-151.

IRIARTE, M.J.; Gómez-Orellana, L.; Muñoz Sobrino, C.; Ramil-Rego, P. y ArRIZABALAgA, A. (2005): La dinámica de la vegetación en el noroeste peninsular durante la transición del paleolítico medio al paleolítico superior. En Montes, R. y Lasheras, J. A. (eds): Neandertales cantábricos. Estado de la cuestión. (Monografias del Museo de Altamira 20) Santander: Museo de Altamira: 231-253.

KoRnPRoBSt, P. Y RAT, P. (1967): Premier résultats d'une étude géologique et paléoclimatique du remplissage paléolithique moyen et supérieur de Lezetxiki (Mondragón, Guipúzcoa), Munibe, 19, 247-260.

LAPLACE, G. y SÁENZ DE BURUAGA, A., 2000: Application de la typologie analytique et structurale à l'étude de l'outillage mousteroïde de L'Abri Olha 2 à Cambo (Kanbo) en Pays Basque. Paléo 12, 261-324.

LAPlACE, G. y SÁEnZ dE BuRUAgA, A., 2002-2003: Typologie analytique et structurale des complexes du Moustérien de la Grotte Gatzarria (Ossas-Suhare, Pays Basque) et de leurs relations avec ceux de I'abri Olha 2 (Cambo, Pays Basque). Pyrenae 33-34, 81-163.

LEROI-GouRHAN, ARL. (1959): Rësultats de l'analyse pollinique de la grotte d'Isturitz, Bulletin de la Société Préhistorique Française, 56, 619624, Paris.

LeRol-Gourhan, ArL. (1984): La place du néandertalien de Saint-Césaire dans la chronologie würmienne, Bull. Soc. Préhist. Française, 81(7) 196-198.

LEROYER, CH. (1987) : Les gisements castelperroniens de Quinçay et de Saint-Césaire: quelques comparaisons préliminaires des études palynologiques, Préhistoire du Poitou-Charente, 125-134, Actes du $111^{\circ}$ congreso de Soc.Savantes, Poitiers 1986.

LLORET, M. Y MAílLO-FERNÁNDEZ, J. M. (2006): Aproximación tecnológica a los niveles 18b, 18c y 16 de la cueva de El Castillo (Puente Viesgo, Cantabria). En En el centenario de la cueva de El Castillo: el ocaso de los Neandertales, edited by V. Cabrera-Valdés, F. Bernaldo de Quirós and J. M. Maíllo-Fernández, pp. 493-512. UNED-CajaCantabria, Santander.

Mailllo-FernándeZ, J. M., 2003: La Transición Paleolítico Medio-Superio en Cantabria. Análisis tecnológico de la industria lítica de Cueva Morín. Ph.D. dissertation, UNED, Madrid.

Maillo-Fernández, J. M. (2006): Archaic Aurignacian lithic technology in Cueva Morin (Cantabria, Spain). In Bar-Yosef, 0. y Zilhão, J. (eds): Towards a definition of Aurignacian. Trabalhos de Arqueologia, 45: 109-128.

MaílLo-FeRnÁndeZ, J. M. (2007): Le Châtelperronien en Espagne: mythes et réalités. Une approche technologique. En Congrès du Centenaire de S.P.F. vol, 3: Un siècle de construction du discours scientifique en Préhistoire: 95-103. 
Maílo-Fernández, J. M.; Valladas, H.; Cabrera Valdés, V. y Bernaldo de Quirós, F., 2001: Nuevas dataciones para el Paleolitico superior de Cueva Morín (Villanueva de Villaescusa, Cantabria). Espacio, Tiempo y Forma 14, 145-150.

Maillo-Fernández, J. M.; CABrera-Valdés, V. y Bernaldo de Quirós, F., 2004. Le débitage lamellaire dans le Moustérien final de Cantabria, Espagne: le cas de El Castillo et Cueva Morin. L'Anthropologie 108, $367-93$.

MARdones, M. Y Jalut, G. (1983) : La tourbière de Biscaye (Alt. 409m. Hautes Pyrénées): approche paléoécologique des 45.000 dernières années, Pollen et Spores, XXV (2), 163-211, Paris.

Martin, P.; Montes, R. y SAnguino, J., 2006: La tecnología lítica del Musteriense final en la región cantábrica: los datos de Covalejos (Velo de Piélagos, Cantabria, España), in En el centenario de la cueva de El Castillo: el ocaso de los Neandertales, eds. V. Cabrera-Valdés, F. Bernaldo de Quirós y J. M. Maillo-Fernández. Santander: UNEDCajaCantabria, 231-248.

MuÑOZ, M.; SÁnChEZ, M. F. y UgaRTE, M. (1989-1990): El entorno geoambiental del yacimiento arqueológico de Kurtzia (Sopela-Barrika, costa occidental de Bizkaia), Munibe, 41, 107-115.

Normand, CH., 2002: Nouvelles données sur l'Aurignacien de la grotte d'Isturitz (commune de Saint-Martin-d'Arberoue ; PyrénéesAtlantiques) à travers I'industrie lithique de la Salle de Saint-Martin. En F. Bon, J. M. Maillo y D. Ortega (eds) : Autour des concepts de Protoaurignacien, d'Aurignacien initial et ancien : unité et variabilité des comportements techniques des premiers groupes d'hommes modernes dans le Sud de la France et le Nord de l'Espagne, Actes de la Table-ronde de Toulouse, 2003. Espacio, Tiempo y Forma, 15: 145-174.

ORTEGA, I. (2004): Cambios y constantes técnicas durante el periodo llamado de Transición. En Neandertales Cantábricos: estado de la cuestión. Preactas de la reunión: 64-65.

PELEGRIN, J., 1995: Technologie Lithique: Le Chôtelperronien de Roc-deCombe (Lot) et de La Côte (Dordogne).Cahiers du Quaternaire 20, pp. 297.
Pike-Tay, A., Cabrera-Valdés, V. y Bernaldo de Quirós, F., 1999: Seasonal variations of the Middle-Upper Paleolithic transition at El Castillo, Cueva Morin and El Pendo (Cantabria, Spain). Journal of Human Evolution 36: 283-317.

Rios, J. (2005): Características de la producción lítica al final del Paleolítico Medio en el País Vasco. El caso del nivel B de Axlor (Dima, Bizkaia). En R Montes y J. A. Lasheras (eds). Neandertales cantábricos. Estado de la cuestión (Monografías del Museo de Altamira 20) Santander: Museo de Altamira: 333-348.

Sáenz de Buruaga, A., 1991: El Paleolítico Superior de la Cueva de Gatzarria, Zuberoa, Pais Vasco. Anejos de Veleia 6, p. 426.

SÁnCHEZ-FERnÁnDEZ, G., 2005: Análisis tecnológico y tipológico del nivel 20e de la cueva de El Castillo (Puente Viesgo, Cantabria). Trabajo de investigación, inédito, UNED.

SÁnchez-Fernández, G. y Maillo-Fernández, J. M., 2006: Soportes laminares en el musteriense final cantábrico: el nivel 20e de la cueva de El Castillo (Cantabria), in Miscelánea en Homenaje a Victoria Cabrera, vol. I, eds. J. M. Maillo-Fernández y E. Baquedano, E. (Zona Arqueológica 7) Madrid: Museo regional de Arqueología-UNED, 264-73.

SÁnCHEZ-GoÑI, M. F. (1993): De la taphonomie pollinique à la reconstitution de l'environment. L'exemple de la region cantabrique, BAR International Series, 586, Oxford

StUCKENRATH, R., 1978: Dataciones de Carbono 14, in Vida y muerte en Cueva Morin, ed. J. González Echegaray y L. G. Freeman. Santander, 215.

TARRIÑo, A. Y NoRmand, CH. (2002): Procedencia de los restos líticos en el Auriñaciense antiguo (C4B1) de Isturitz (Pyrénées-Atlantiques, Francia). In Autour des concepts de Protoaurignacien, d'Aurignacien archaïque, initial et ancien, edited by F. Bon, J. M. Maillo-Fernández and D. Ortega, pp. 67-86. Espacio, Tiempo y Forma 15, Madrid.

Vega del Sella, Conde de LA, 1921: El Paleolítico de Cueva Morín (Santander) y notas para la climatología cuaternaria. Madrid: Comisión de Investigaciones Paleontológicas y Prehistóricas 29. 